\title{
Randomised controlled trial of telemedicine for new neurological outpatient referrals
}

\author{
R Chua, J Craig, R Wootton, V Patterson
}

\begin{abstract}
Objective-To test the hypothesis that telemedicine for new patient referrals to neurological outpatients is as efficient and acceptable as conventional face to face consultation.
\end{abstract}

Methods-A randomised controlled trial between two groups: face to face (FF) and telemedicine (TM). This study was carried out between a neurological centre and outlying clinics at two distant hospitals linked by identical medium cost commercial interactive video conferencing equipment with ISDN lines transmitting information at $384 \mathrm{kbits} / \mathrm{s}$. The same two neurologists carried out both arms of the study.

Of the 168 patients who were suitable for the study, 86 were randomised into the telemedicine group and 82 into the face to face group. Outcome measures were (1) consultation process: (a) number of investigations; (b) number of drugs prescribed; (c) number of patient reviews and (2) patient satisfaction: (a) confidence in consultation; (b) technical aspects of consultation; (c) aspects surrounding confidentiality. Diagnostic categories were also measured to check equivalence between the groups: these were structural neurological, structural non-neurological, nonstructural, and uncertain.

Results-Diagnostic categories were similar $(p>0.5)$ between the two groups. Patients in the telemedicine group had significantly more investigations $(p=0.001)$. There was no difference in the number of drugs prescribed $(p>0.5)$. Patients were generally satisfied with both types of consultation process except for concerns about confidentiality and embarrassment in the telemedicine group $(p=0.017$ and $p=0.005$ respectively).

Conclusion-Telemedicine for new neurological outpatients is possible and feasible but generates more investigations and is less well accepted than face to face examination.

(F Neurol Neurosurg Psychiatry 2001;71:63-66)

Keywords: telemedicine; neurology; randomised controlled trial

Health, University of

Queensland, Brisbane,

Australia

R Wootton

Correspondence to:

Dr R Chua

tele.neuro@royalhospitals.

n-i.nhs.uk

Received 8 November 2000 and in revised form

23 February 2001

Accepted 29 February 2001 a resident neurologist. Using telemedicine a neurologist is not only able to take a history from the patient but also witness and direct the neurological examination. So far we have shown that it is possible to conduct a neurological examination using such a system, ${ }^{1}$ and that it is feasible, safe, and satisfactory to both the doctors and patients. ${ }^{2-4}$ These findings should be broadly applicable to neurological outpatients as the process of history taking and examination is identical.

In the United Kingdom neurologists are mainly based in large tertiary referral hospitals. ${ }^{5}$ Within the clinical setting of the neurological outpatients, the neurologist either travels to the distant general hospital or the patients travel to the neurological centre. This may involve considerable distances and result in either inefficient use of the specialist's or patient's time.

In a recent pilot study we showed that it was feasible and safe for a neurologist to assess new referrals to a neurological outpatient clinic using telemedicine. ${ }^{6}$ Here we conducted a randomised controlled study to compare telemedicine with conventional face to face consultation for measures of process and patient satisfaction, to test the hypothesis that there would be little difference between the two methods.

\section{Methods}

HOSPITALS INVOLVED IN THE STUDY

This study was carried out between two small hospitals and the neurological centre based in the Royal Victoria Hospital, Belfast. Both the Tyrone County Hospital, Omagh and the Erne Hospital, Enniskillen are rural hospitals administered by the Sperrin Lakeland Hospital and Community Trust serving a population of 60 000 and 57000 respectively. The distances between the Tyrone County Hospital and Erne Hospital from the neurological centre are 70 and 85 miles and the round trip time 3 and 3.5 hours respectively. There are no neurologists based in either of them but at the time of the study the Tyrone County Hospital had a visiting neurologist twice a month and Erne Hospital once a month.

\section{TELEMEDICINE CONSULTATION}

Identical medium cost commercial interactive video conferencing equipment (PCS P100P roll-about processor, PCS C300P camera unit, PCS A500 audiounit, Sony) were installed between the neurological centre and the two rural hospitals. They were connected by three ISDN lines transmitting information at $384 \mathrm{kbits} / \mathrm{s}$. 


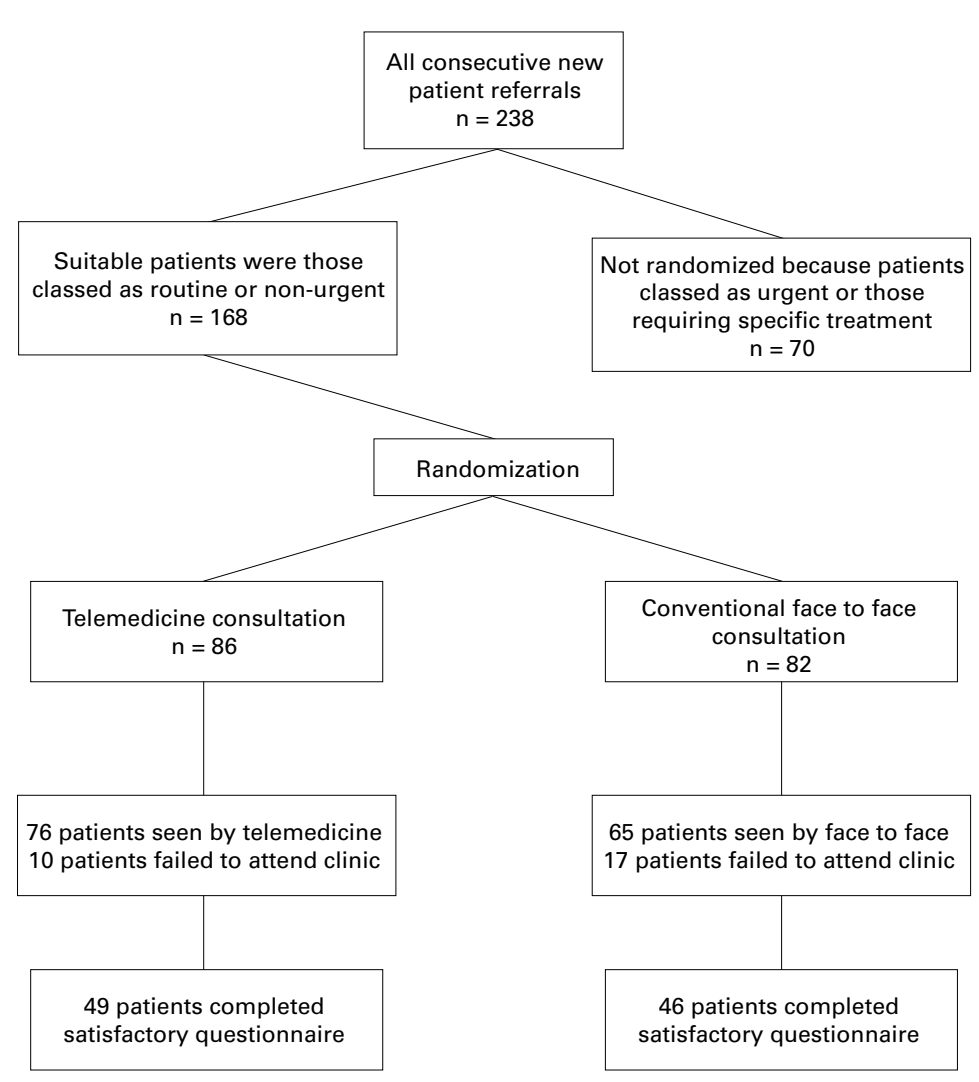

Figure 1 Flow chart summarising the progress of study recruits.

PATIENT SELECTION AND RANDOMISATION

All consecutive patients who were referred by their general practitioner to either rural hospital for a new neurological outpatient consultation and whose referral letter was graded as non-urgent by the consultant neurologist were eligible for the study. Exclusion criteria were patients who were classed as urgent or those referred for a specific treatment such as botulinum toxin injection. Patients were stratified by their presenting symptoms and time on the waiting list. They were then randomised to either face to face or telemedicine consultation based on the last digit of their hospital number (odd numbers to telemedicine and even numbers to face to face) so that each group had a roughly equal number of similar symptoms (headaches, weakness, sensory disturbance, dizziness, alteration of consciousness, tremor, and miscellaneous).

Table 1 Demographic data of patients

\begin{tabular}{llll}
\hline & $F F$ & $T M$ & $p$ Value \\
\hline Number of patients & 65 & 76 & \\
Mean age (y) & 43.7 & 41.8 & 0.48 \\
Range of age (y) & $16-78$ & $15-83$ & 0.76 \\
Mean time to consultation (weeks) & 42.7 & 44 & \\
Sex distribution (n(\%)): & $38(58.5)$ & $38(50)$ & 0.31 \\
$\quad$ Males & $27(41.5)$ & $38(50)$ & \\
$\quad$ Females & $24(36.9)$ & $26(34.2)$ & \\
Diagnostic category (n(\%)): & $3(4.6)$ & $3(3.9)$ & 0.77 \\
SN & $33(50.8)$ & $37(48.7)$ & \\
SNN & $5(7.7)$ & $10(13.2)$ & \\
NS & &
\end{tabular}

$\mathrm{FF}=$ Face to face consultation; $\mathrm{TM}=$ telemedicine consultation; $\mathrm{SN}=$ structural neurological; $\mathrm{SNN}=$ structural non-neurological; $\mathrm{NS}=$ non-structural; $\mathrm{U}=$ uncertain.
TELEMEDICINE AND FACE TO FACE PROCESS

The two neurologists who were involved in the study were a consultant (VP) and a neurological trainee (JC, specialist registrar 4). All patients in the face to face and telemedicine groups were seen by one of the two neurologists. For the telemedicine group a neurology research registrar (RC) travelled to the rural hospitals where before the patient consultation he summarised the patients' history and all relevant details using telemedicine and then examined the patients' optic fundi. Before the patients were brought into the consultation room they were informed about the objectives and processes of the teleconsultation.

The neurologist then interviewed the patient and any other key witnesses available by telemedicine. The research registrar then carried out a standardised neurological examination and performed any other relevant examination and these were directed and witnessed by the distant neurologist. When this was completed any relevant radiological images were viewed by focusing the camera onto the films placed on the $x$ ray viewing box in the clinical room. The neurologist then discussed the diagnosis and outlined the management plan to the patients and, where appropriate, carers.

\section{OUTCOME MEASURES}

\section{Consultation process}

The outcome measures used to compare the processes of consultation between the face to face and telemedicine groups were modified from those used in a previous neurological study of outpatients. ${ }^{7}$ To determine equivalence between the groups we also recorded time to consultation (from appointment letter sent), diagnostic category (structural neurological, structural non-neurological, nonstructural, or uncertain), actions taken after consultation (investigations ordered, referrals made, and treatment prescribed), and disposal method (discharged after first appointment, followed up at neurological outpatients, or admitted to hospital). Investigations were categorised as neurological MRI, CT, EEG, EMG, nerve conduction studies (NCS), Doppler scan, non-neurological blood tests, chest radiography, and cardiological investigations.

\section{Patient satisfaction}

All patients in both groups were sent an identical self administered questionnaire which consisted of three point Likert-type scales relating to eight statements (tables 4 and 5). These related to aspects of confidence in the consultation; technical aspects of the consultation process, and aspects surrounding confidentiality. Space for additional comments was available.

\section{DATA ANALYSIS}

The data between the two groups were analyzed using the $\mathrm{Z}$ test for parametric data, $\chi^{2}$ test for categorical data, and the MannWhitney $U$-Wilcoxon rank sum test for nonparametric continuous variables. 
Table 2 List of structural neurological disease seen

\begin{tabular}{lcl}
\hline Structural neurological disease & FF & TM \\
\hline Epilepsy & 10 & 7 \\
Peripheral neuropathy & 3 & 3 \\
Benign essential tremor & 2 & 4 \\
Transient ischaemic attack & 2 & 1 \\
Previous head injury & 0 & 2 \\
Cerebellar disease & 1 & 1 \\
Myasthenia gravis & 1 & 0 \\
Previous encephalitis & 1 & 1 \\
Multiple sclerosis & 1 & 1 \\
Hydrocephalus & 1 & 0 \\
Cervical radiculopathy & 1 & 0 \\
Parkinson's disease & 1 & 0 \\
Cataplexy & 0 & 1 \\
Reflex anoxic seizure & 0 & 1 \\
Senile ptosis & 0 & 1 \\
Periodic limb movements & 0 & 1 \\
Reflex sympathetic dystrophy & 0 & 1 \\
Motor neuron disease & 0 & 1
\end{tabular}

$\mathrm{FF}=$ Face to face consultation; $\mathrm{TM}=$ telemedicine consultation.

\section{Results}

Of the 168 new patients who were randomised to the study ( 82 to face to face and 86 to telemedicine), the two neurologists between them saw 141 patients ( 65 by face to face and 76 by telemedicine) with the remainder not keeping their appointments. The flow chart summarising the progress of the patients is given in figure 1. The characteristics of the two groups are summarised in table 1 and the types of structural disease in table 2. The measures of process for the two groups are shown in table 3.

Although there was roughly an equal proportion of patients who had structural disease in each group, patients who were seen by telemedicine had significantly more investigations $(\mathrm{p}=0.001)$. Prescription numbers did not differ between the patients who were seen in either group $(p>0.5)$. There were slightly more patients given a further neurological review appointment after the first consultation in the telemedicine group (29\%) compared with the face to face group $(22 \%)(\mathrm{p}=0.42)$. No patients required immediate admission to the neurological inpatient unit in either group and one patient was referred to another speciality in the face to face group.

Ninety five patients $(67 \%)$ in the study completed their satisfaction questionnaire. Table 4 summarises patient satisfaction and confidence in the consultation processes between the two groups (statements 1 to 5 ). Table 5 summarises the responses to the technical aspect and issues of confidentiality (statements 6 to 8).

Overall, patients were satisfied and had confidence in the consultations. There were no differences between the two groups for statements $1,2,4$, and $5(\mathrm{p}>0.5)$ but fewer patients agreed with statement $3(p=0.02)$ in the telemedicine group. Likewise, there was no difference in the technical aspects of communica-

Table 3 Measures of process

\begin{tabular}{lllc}
\hline & $F F$ & $T M$ & $p$ Value \\
\hline Number of investigations: & 6 & 26 & 0.001 \\
$\quad$ Neurological & 5 & 20 & $>0.5$ \\
$\quad$ Non-neurological & $11(16.9 \%)$ & $14(18.4 \%)$ & $>0.42$ \\
$\quad$ Prescription numbers & $51(78.5 \%)$ & $54(71.1 \%)$ & 0 \\
Disposal: & & &
\end{tabular}

$\mathrm{FF}=$ Face to face consultation; $\mathrm{TM}=$ telemedicine consultation.
Table 4 Patient satisfaction and confidence in the consultation process

\begin{tabular}{|c|c|c|c|}
\hline Statement & $F F$ & $T M$ & $p$ Value \\
\hline \multicolumn{4}{|c|}{ (1) I was able to say what I wanted: } \\
\hline Disagree & 11 & 11 & \multirow{3}{*}{0.97} \\
\hline No opinion & 3 & 4 & \\
\hline Agree & 32 & 34 & \\
\hline \multicolumn{4}{|c|}{ (2) I felt the neurologist understood my problems: } \\
\hline Disagree & 10 & 10 & \multirow{3}{*}{0.97} \\
\hline No opinion & 4 & 5 & \\
\hline Agree & 32 & 34 & \\
\hline \multicolumn{4}{|c|}{ (3) I felt the explanation of my symptoms was satisfactory: } \\
\hline Disagree & 8 & 13 & \multirow{3}{*}{0.02} \\
\hline No opinion & 2 & 9 & \\
\hline Agree & 36 & 27 & \\
\hline \multicolumn{4}{|c|}{ (4) I felt the outpatient appointment was useful: } \\
\hline Disagree & 8 & 10 & \multirow{3}{*}{0.53} \\
\hline No opinion & 5 & 7 & \\
\hline Agree & 33 & 32 & \\
\hline \multicolumn{4}{|c|}{$\begin{array}{l}\text { (5) I had confidence in the way the neurologist addressed my } \\
\text { problems: }\end{array}$} \\
\hline Disagree & 8 & 10 & \multirow{3}{*}{0.65} \\
\hline No opinion & 6 & 7 & \\
\hline Agree & 32 & 32 & \\
\hline
\end{tabular}

$\mathrm{FF}=\mathrm{Face}$ to face consultation; $\mathrm{TM}=$ telemedicine consultation.

Table 5 Patient satisfaction in the technical aspect and confidentiality

\begin{tabular}{lccc}
\hline Statement & $F F$ & $T M$ & p Value \\
\hline (6) I felt shy and nervous about speaking: & 33 & 21 & \\
Disagree & 4 & 7 & 0.005 \\
No opinion & 9 & 21 & \\
Agree & 2 & 7 & \\
(7) I could hear everything the neurologist said: & \\
Disagree & 1 & 2 & 0.08 \\
No opinion & 43 & 40 & \\
Agree & 35 & 28 & \\
(8) I was worried that others were listening & & \\
Disagree & 10 & 9 & 0.017 \\
No opinion & 1 & 12 & \\
Agree & & & \\
\hline
\end{tabular}

$\mathrm{FF}=\mathrm{Face}$ to face consultation; $\mathrm{TM}=$ telemedicine consultation.

tion between the groups ( $p>0.05)$. However, statements comparing embarrassment and discomfort and perceptions of confidentiality using telemedicine showed significant differences between the two groups $(p=0.005$ and $\mathrm{p}=0.017$ respectively).

\section{Discussion}

In this randomised controlled trial every single measure of process and satisfaction favoured the face to face group. The significant difference in process was more investigations in the telemedicine group (60 per 100 patients telemedicine $v 17$ per 100 patients face to face) and in three out of the eight satisfaction questions the differences were statistically significant. It is most likely that the process of telemedicine was associated with less diagnostic certainty and therefore generated more investigations. It was not due to differences in practice between the two neurologists involved or the waiting time. The groups seemed well matched for diagnostic categories so there was no excess of structural neurological disease in the telemedicine group to explain the differences, even though the investigation numbers in the face to face group seem low. The concern about confidentiality and embarrassment in the telemedicine group could be lessened by a more thorough explanation of the teleconsultation process.

The strengths of this study are that it was conducted in a real life clinical setting and that 
its results are liable to be widely applicable. It is the largest study of telemedicine for neurological outpatients and the only randomised study.

One of its weaknesses was the exclusion of urgent patient referrals. These, however, have many similarities to emergency neurological admissions, a group in which we have shown telemedicine to be safe and effective. ${ }^{38} \mathrm{We}$ used a neurological research fellow at the distant end to examine the patients. This was done for organisational reasons and again we know from our inpatient work that doctors with 1 to 3 years experience are capable of performing the required examination and are satisfied with it. ${ }^{1}$ We also did not calculate the projected number for adequate sample size because there is so little available information on conventional management of neurological outpatients. Fortunately the size was sufficient to enable us to reject our hypothesis.

The results are likely to be widely applicable. Although we have shown that telemedicine is less efficient and well received by patients than face to face examination, it is still a perfectly possible way of carrying out a neurological consultation and the results of telemedicine consultation may well be within the range of face to face practice of various neurologists. In practical terms telemedicine may be the only feasible way of carrying out a consultation-for example, during a transport strike. In economic terms telemedicine saves the time of the neurologist and where the cost of the neurologist is high it may be economically preferable. This will be the subject of a future analysis.

We thank the Research and Development Office of the Northern Ireland Department of Health and Social Services for their financial support and Queens University Belfast for loan of equipment.

1 Craig JJ, McConville JP, Patterson VH, et al. Neurological examination is possible using telemedicine. I Telemed Telecare 1999;5:177-81.

2 Craig JJ, McConville JP, Patterson VH, et al. Teleneurology: possibilities of improving patient care. Neurology 1999;52: (suppl 2):143-4.

3 Craig JJ, Russell C, Patterson VH, et al. Telemedicine is a safe way of managing inpatients with neurological probsafe way of managing inpatients with

4 Craig JJ, Russell C, Patterson VH, et al. User satisfaction Craig JJ, Russell C, Patterson VH, et al. User satisfaction
with real-time teleneurology. F Telemed Telecare 1999;5: 237-42.

5 Cartlidge N, Stevens D, Johnson M, et al. Neurology in the UK. Towards 2000 and beyond. London: ABN, 1997.

6 Craig JJ, Chua R, Wootton R, et al. A pilot study of telemedicine for new neurological outpatient referrals. $\mathcal{f}$ Telemed Telecare 2000;6:225-8.

7 Patterson VH, Esmonde TFG. Comparison of the handling of neurological outpatient referrals by general physicians and a neurologist. F Neurol Neurosurg Psychiatry 1993;56: $830-3$.

8 Patterson V, Craig J, Pang KA, et al. Successful management of unexplained coma by telemedicine. I Telemed Telecare 1999;5:134-6. 